\title{
ON THE BIADJOINT OF RIESZ-LIKE HOMOMORPHISMS ON PARTIALLY ORDERED VECTOR SPACES
}

\author{
Gerard Buskes and Jamie Summerville
}

We generalise to partially ordered vector spaces, with a new technique, Arendt's approach to Kim's characterisation of Riesz homomorphisms.

In [8] Kim proved the elegant duality connection between Riesz homomorphisms and interval preserving maps. A particular consequence is the fact, referred to as Kim's Theorem in this paper, that the biadjoint of a Riesz homomorphism is again a Riesz homomorphism. It is the latter consequence that we focus on. Kim's Theorem has interesting applications in spectral theory and there is a need for similar results in the much wider context of partially ordered vector spaces. Two such results exist in the literature, one by Wickstead [14] and one by Takeo [12] (also see [13]). Their theorems are in the setting of partially ordered Banach spaces with the Riesz decomposition property and therefore do not generalise Kim's Theorem. Motivated by Arendt's proof of Kim's Theorem in [2] (also see [1, Theorem 7.4]) as well as by the notion of enveloping Riesz space in [3] (also see [5]), we arrive in this paper at general purely order theoretic results for partially ordered vector spaces. Thus, our Theorem 13 generalises Kim's Theorem. Proposition 11 and Theorems 4 and 8 expand the corresponding theorems by Arendt in [2] to partially ordered vector spaces. Lemma 5, in the case of Riesz spaces, can be found as [1, Exercise 1, p.28]. Our notation and terminology will be standard and we refer to $[1,10,11,15]$. We first make a blanket assumption. Throughout this paper,

$E, F$ and $G$ are Archimedean, directed, partially ordered vector spaces.

We denote by $L_{r}(E, F)$ the directed partially ordered vector space of all regular operators $E \rightarrow F$, that is, the space of all differences of positive operators $E \rightarrow F$. Instead of $L_{r}(E, \mathbf{R})$ we write $E^{\sim}$. A map $T: E \rightarrow F$ is interval preserving if $T$ is positive and for every $x \in E^{+}$and $y \in F^{+}$with $y \leqslant T(x)$, there exists $z \in E^{+}$such that $z \leqslant x$ and $T(z)=y$. The choice of an analogue for Riesz homomorphism in the setting of partially ordered vector spaces is not obvious at all. Somewhat surprisingly, the choice of homomorphisms that was made in [3] to describe the enveloping Riesz space does not work well for this duality problem as we shall show in the example following Theorem 10. Instead we have opted for a stronger type of homomorphism that is very

Received 3rd February 1997.

Copyright Clearance Centre, Inc. Serial-fee code: 0004-9729/97 \$A2.00+0.00. 
close to Wickstead's definition in [14]. If $F$ is a Riesz space, we say that a positive operator $T: E \rightarrow F$ is an $R$-homomorphism if for all $x, y \in E$ there exists $z \geqslant x, y$ in $E$ for which

$$
T(z)=T(x) \vee T(y),
$$

or equivalently, if for all $x, y \in E^{+}$there exists $z \leqslant x, y$ in $E$ for which $T(z)=T(x) \wedge T(y)$. This R-homomorphism coincides with Wickstead's definition in [14] (which originated in $[6]$ ), when one restricts the range space to be a Riesz space in his definition. In case that $E$ is a Riesz space as well, the class of R-homomorphisms coincides with the class of Riesz homomorphisms. Every R-homomorphism is positive. For a regular map $T: E \rightarrow F$ we define the (regular) adjoint $T^{\sim}: F^{\sim} \rightarrow E^{\sim}$ by $T^{\sim}(\phi)(e)=\phi(T(e))$ for all $\phi \in F^{\sim}$ and $e \in E$. Finally, as is common convention, a map between two vector spaces is called sublinear if it is positive homogeneous and subadditive. One of our goals is a proof of the following result.

THEOREM 1. Let $G$ be a Riesz space. If $T: E \rightarrow G$ is an $R$-homomorphism then $T^{\sim \sim}$ is an R-homomorphism $E^{\sim \sim} \rightarrow G^{\sim \sim}$.

A powerful tool in the proof of this theorem will be the following Mazur-Orlicz type sandwich theorem (see [10, Theorem 1.5.3] for a proof).

Theorem 2. Let $F$ be a Dedekind complete Riesz space. Let $p: E \rightarrow F$ be a sublinear map, $A \subset E$ nonempty and convex, and $q: A \rightarrow F$ concave (that is, $q(t x+(1-t) y) \geqslant t q(x)+(1-t) q(y)$ for all $x, y \in A$ and $0 \leqslant t \leqslant 1)$ with $q \leqslant p$ on $A$. Then there exists a linear operator $T: E \rightarrow F$ such that $T \leqslant p$ everywhere, and $q \leqslant T$ on $A$.

Our avenue for proving Theorem 1 will be a wide generalisation of the following result by Arendt (see [2] or [1, Theorem 7.4]).

Theorem 3. (Arendt) Let $E, G$ and $F$ be Riesz spaces with $F$ also Dedekind complete. Let $T: E \rightarrow G$ be a linear operator.

(i) If $T$ is a Riesz homomorphism, then the map $S \longmapsto S \circ T$ from $L_{r}(G, F)$ into $L_{r}(E, F)$ is interval preserving.

(ii) If $T$ is interval preserving, then the map $S \longmapsto S \circ T$ from $L_{r}(G, F)$ into $L_{r}(E, F)$ is a Riesz homomorphism.

Our version of part (i) for partially ordered vector spaces (see Theorem 4 below) involves an argument in which Arendt's Theorem itself will be used. The analogue of (ii) above requires, however, a different and more complicated approach. At the same time we wish to use the fact that (ii) still holds, with exactly the same proof as provided in [1], in case that $E$ and $G$ merely have the Riesz decomposition property. The first step in the direction of Theorem 1 is the following result. 
THEOREM 4. Let $G$ be a Riesz space, and suppose that $F$ is a Dedekind complete Riesz space. If $T: E \rightarrow G$ is an $R$-homomorphism then the map $S \longmapsto S \circ T$ from $L_{r}(G, F)$ into $L_{r}(E, F)$ is interval preserving.

Proof: Note that $L_{r}(G, F)$ is a Dedekind complete Riesz space (see [7]), while $L_{r}(E, F)$ is an Archimedean, directed, partially ordered vector space (not necessarily a Riesz space). Let $T: E \rightarrow G$ be an R-homomorphism. The map $S \longmapsto S \circ T$ from $L_{r}(G, F)$ into $L_{r}(E, F)$ obviously is positive. Fix $S \in L_{r}(G, F)$ with $S \geqslant 0$, and let $V$ in $L_{r}(E, F)$ be such that $0 \leqslant V \leqslant S \circ T$. We shall show the existence of $S_{1} \in L_{r}(G, F)$ with $0 \leqslant S_{1} \leqslant S$ and $S_{1} \circ T=V$.

$E$ and $G$, being Archimedean, have Dedekind completions $E^{\delta}$ and $G^{\delta}$ respectively (see $\left[11,1.19\right.$, p.151]). Moreover, $E$ is a majorising vector subspace of $E^{\delta}$, and it follows from [4, Corollary 2.7] that $T$ can be extended to a Riesz homomorphism $T^{\delta}: E^{\delta} \rightarrow G^{\delta}$. By Kantorovič's Theorem (see [1, Theorem 2.8]) it follows that $S$ can be extended to a positive $S^{\delta}: G^{\delta} \rightarrow F$.

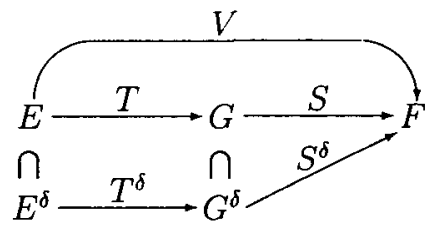

For all $x \in E^{\delta}$, define

$$
p(x)=\left(S^{\delta} \circ T^{\delta}\right)\left(x^{+}\right)
$$

where $x^{+}$is in $E^{\delta}$. It is elementary to show that $p$ is sublinear. To prove that $p$ dominates $V$, let $x \in E$. Since $T^{\delta}$ is a Riesz homomorphism,

$$
p(x)=\left(S^{\delta} \circ T^{\delta}\right)\left(x^{+}\right)=S^{\delta}\left(T^{\delta}(x)^{+}\right),
$$

where again, $x^{+}$is in $E^{\delta}$. By the definition of R-homomorphism, there exists $h_{x} \in E^{+}$ with $h_{x} \geqslant x$ and $T\left(h_{x}\right)=T(x)^{+}$. Thus,

$$
V(x) \leqslant V\left(h_{x}\right) \leqslant(S \circ T)\left(h_{x}\right)=S^{\delta}\left(T\left(h_{x}\right)\right)=S^{\delta}\left(T(x)^{+}\right)=S^{\delta}\left(T^{\delta}(x)^{+}\right)=p(x) .
$$

By the Hahn-Banach Theorem (see [1, Theorem 2.1]), there exists a linear $V^{\delta}: E^{\delta} \rightarrow$ $F$ with $\left.V^{\delta}\right|_{E}=V$ and $V^{\delta}(x) \leqslant p(x)$ for all $x$ in $E^{\delta}$. Because $V^{\delta}(-x) \leqslant p(-x)=$ $\left(S^{\delta} \circ T^{\delta}\right)\left((-x)^{+}\right)=0$ for every $x \in E^{\delta+}$, it follows that $V^{\delta} \geqslant 0$. Furthermore, for every $x \in E^{\delta+}$ we have

$$
V^{\delta}(x) \leqslant p(x)=\left(S^{\delta} \circ T^{\delta}\right)\left(x^{+}\right)=\left(S^{\delta} \circ T^{\delta}\right)(x)
$$

By part (i) of Arendt's Theorem above, there exists $S_{1}^{\delta} \in L_{r}\left(G^{\delta}, F\right)$ such that $0 \leqslant S_{1}^{\delta} \leqslant$ $S^{\delta}$ and $S_{1}^{\delta} \circ T^{\delta}=V^{\delta}$. Defining $S_{1}=\left.S_{1}^{\delta}\right|_{G}$, we obtain

$$
V=\left.V^{\delta}\right|_{E}=\left.\left(S_{1}^{\delta} \circ T^{\delta}\right)\right|_{E}=S_{1}^{\delta} \circ T=S_{1} \circ T
$$


and

$$
S_{1}=\left.S_{1}^{\delta}\right|_{G} \leqslant\left. S^{\delta}\right|_{G}=S
$$

To arrive at an analogous result for part (ii) of Arendt's Theorem we have to work harder. Before we can present such an analogue, we need three lemmas which are of interest in their own right.

LEMMA 5. Let $E$ be a solid, directed, partially ordered vector subspace of $G$ with the Riesz decomposition property. Let $F$ be a Dedekind complete Riesz space. Then the map $R: L_{r}(G, F) \rightarrow L_{r}(E, F)$ defined by $R(T)=\left.T\right|_{E}$ is an R-homomorphism. In particular, if $G$ has the Riesz decomposition property as well, then $R$ is a Riesz homomorphism between the Dedekind complete Riesz spaces $L_{r}(G, F)$ and $L_{r}(E, F)$.

Proof: $R$ is obviously positive. To show that $R$ is an R-homomorphism we need to find for every $S, T \in L_{r}(G, F)$ with $S, T \geqslant 0$ an $H \in L_{r}(G, F)$ such that $H \leqslant S, T$ and $R(H)=R(S) \wedge R(T)$. Let $S, T \in L_{r}(G, F)$ with $S, T \geqslant 0$. Since $L_{r}(G, F)$ is not necessarily a Riesz space, the infimum of $\{S, T\}$ may not exist. Instead, we define $T \sqcap S$ on $G^{+}$by

$$
(T \sqcap S)(x)=\inf \left\{T(u)+S(v): u, v \in G^{+} \text {and } u+v=x\right\} .
$$

By a slight abuse of language, but with a straightforward proof, $T \sqcap S$ is sublinear on $G^{+}$. From here the proof proceeds in two steps.

STEP 1. $T \sqcap S$ is linear on $E^{+}$.

Let $x \in E^{+}$. In $L_{r}(E, F)$, which is a Riesz space, we have

$$
(R(T) \wedge R(S))(x)=\inf \left\{T(u)+S(v): u, v \in E^{+} \text {and } u+v=x\right\} .
$$

Obviously, $\inf \left\{T(u)+S(v): u, v \in E^{+}, u+v=x\right\} \geqslant \inf \left\{T(u)+S(v): u, v \in G^{+}\right.$, $u+v=x\}$. To obtain the opposite inequality, let $u, v \in G^{+}$with $u+v=x$. Then $0 \leqslant u \leqslant x$ and $0 \leqslant v \leqslant x$, and since $E$ is a solid subspace of $G$ it follows that $u, v \in E^{+}$. Hence, $\left.(T \sqcap S)\right|_{E^{+}}=\left.(R(T) \wedge R(S))\right|_{E^{+}}$.

STEP 2: There exists an $H$ as desired.

Define the sublinear $p: G \rightarrow F$ by

$$
p(x)=\inf \left\{(T \sqcap S)(y): y \in G^{+} \text {and } y \geqslant x\right\} .
$$

Note that $T \sqcap S$ is monotone, since $S, T \geqslant 0$. Thus, for every $x \in G^{+}$,

$$
0 \leqslant p(x)=\inf \left\{(T \sqcap S)(y): y \in G^{+} \text {and } y \geqslant x\right\}=(T \sqcap S)(x) \text {. }
$$

By the sandwich Theorem 2, there exists a linear operator $H: G \rightarrow F$ such that $H \leqslant p$ on $G$ and $\left.(T \sqcap S)\right|_{E^{+}} \leqslant\left. H\right|_{E^{+}}$. This implies that for every $x \in E^{+},(T \sqcap S)(x) \leqslant H(x) \leqslant$ $p(x) \leqslant(T \sqcap S)(x)$. Hence, $H \in L_{r}(G, F)^{+}$and $\left.H\right|_{E^{+}}=\left.(T \sqcap S)\right|_{E^{+}}=\left.(R(T) \wedge R(S))\right|_{E^{+}}$. Therefore, $R(H)=R(T) \wedge R(S)$ and $H \leqslant S, T$. 
In the following proposition we show that the composition of R-homomorphisms again is an $\mathrm{R}$-homomorphism. We remind the reader of $[3$, Example 1.10] where the composition of similar but weaker Riesz-like homomorphisms is less pleasant.

Lemma 6. The composition of two R-homomorphisms is an R-homomorphism.

Proof: Let $G$ and $F$ be Riesz spaces. Let $T_{1}: G \rightarrow F$ and $T_{2}: E \rightarrow G$ be Rhomomorphisms. Then $T_{1}$ is a Riesz homomorphism, since $G$ and $F$ are Riesz spaces. Of course $T_{1} \circ T_{2}$ is a positive map. Let $x, y \in E$. Since $T_{2}$ is an $\mathrm{R}$-homomorphism, there exists $h \in E$ with $h \geqslant x, y$ and $T_{2}(h)=T_{2}(x) \vee T_{2}(y)$. Thus,

$$
\left(T_{1} \circ T_{2}\right)(x) \vee\left(T_{1} \circ T_{2}\right)(y)=T_{1}\left(T_{2}(x) \vee T_{2}(y)\right)=\left(T_{1} \circ T_{2}\right)(h)
$$

Therefore, $T_{1} \circ T_{2}$ is an R-homomorphism.

Lemma 7 . Let $E$ have the Riesz decomposition property. If $T: E \rightarrow G$ is interval preserving then $T(E)$ is a solid, directed, partially ordered vector subspace of $G$. In addition, $T(E)$ has the the Riesz decomposition property.

Proof: The directedness of $T(E)$ is immediate. To prove that $T(E)$ is solid in $G$, let $y \in G$ and $x \in T(E)$ such that $0 \leqslant y \leqslant x$. Because $T$ is interval preserving there exists $x_{0} \in E$ with $0 \leqslant x_{0} \leqslant x$ and $T\left(x_{0}\right)=y$. Thus $y \in T(E)$.

For the Riesz decomposition property of $T(E)$ we reason as follows. Let $y, z_{1}, z_{2} \in$ $T(E)$ with $z_{1}, z_{2} \geqslant 0$ and $0 \leqslant y \leqslant z_{1}+z_{2}$. Since $z_{1} \in T(E)$ there exists $y_{0} \in E$ such that $T\left(y_{0}\right)=z_{1}$. Because $E$ is directed there exists $y_{1}, y_{2} \in E^{+}$with $y_{0}=y_{1}-y_{2}$. Then $z_{1}=T\left(y_{0}\right)=T\left(y_{1}\right)-T\left(y_{2}\right)$, where $T\left(y_{1}\right)$ and $T\left(y_{2}\right)$ are positive since $T$ is positive. Then $0 \leqslant z_{1} \leqslant T\left(y_{1}\right)$. Because $T$ is interval preserving there exists $z_{1}^{\prime} \in E$ with $0 \leqslant z_{1}^{\prime} \leqslant y_{1}$ and $T\left(z_{1}^{\prime}\right)=z_{1}$. Similarly, there exists $z_{2}^{\prime} \in E$ with $0 \leqslant z_{2}^{\prime}$ and $T\left(z_{2}^{\prime}\right)=z_{2}$. Because $T$ is interval preserving, we can find $x \in E$ with $0 \leqslant x \leqslant z_{1}^{\prime}+z_{2}^{\prime}$ and $T(x)=y$. Since $E$ has the Riesz decomposition property there exists $x_{1}^{\prime}, x_{2}^{\prime} \in E$ with $0 \leqslant x_{1}^{\prime} \leqslant z_{1}^{\prime}, 0 \leqslant x_{2}^{\prime} \leqslant z_{2}^{\prime}$, and $x=x_{1}^{\prime}+x_{2}^{\prime}$. By the positivity of $T$ we have that $y=T\left(x_{1}^{\prime}\right)+T\left(x_{2}^{\prime}\right)$ and $0 \leqslant T\left(x_{1}^{\prime}\right) \leqslant z_{1}$ and $0 \leqslant T\left(x_{2}^{\prime}\right) \leqslant z_{2}$.

We are now in a position to prove the analogue to part (ii) of Arendt's Theorem.

THEOREM 8. Let $E$ have the Riesz decomposition property. Assume furthermore that $F$ is Dedekind complete. If $T: E \rightarrow G$ is interval preserving, then the map $S \longmapsto S \circ T$ from $L_{r}(G, F)$ into $L_{r}(E, F)$ is an $R$-homomorphism.

Proof: Define $T_{1}: L_{r}(G, F) \rightarrow L_{r}(E, F)$ by $T_{1}(S)=\left.S\right|_{T(E)}$. By the previous lemma, $T(E)$ is directed, has the Riesz decomposition property, and is solid in $G$. Lemma 5 implies that $T_{1}$ is an R-homomorphism. Define $T_{2}: L_{r}(T(E), F) \rightarrow L_{r}(E, F)$ by $T_{2}(S)=S \circ T$. As observed right after Theorem 3, a variation of (ii) in Arendt's Theorem yields that $T_{2}$ is a Riesz homomorphism. 
Consider $T_{2} \circ T_{1}: L_{r}(G, F) \rightarrow L_{r}(E, F)$. Lemma 6 implies that $T_{2} \circ T_{1}$ is an $\mathrm{R}$ homomorphism. For every $S \in L_{r}(G, F)$ we have,

$$
\left(T_{2} \circ T_{1}\right)(S)=T_{2}\left(\left.S\right|_{T(E)}\right)=\left(\left.S\right|_{T(E)}\right) \circ T=S \circ T .
$$

From Theorem 4 (respectively Theorem 8 ) we now derive results on the adjoint of an R-homomorphism and (respectively) the adjoint of an interval preserving map.

Theorem 9. Let $G$ be a Riesz space. If $T: E \rightarrow G$ is an R-homomorphism then $T^{\sim}: G^{\sim} \rightarrow E^{\sim}$ is interval preserving.

ProOF: For every $\varphi \in E^{\sim}$, we have that $T^{\sim}(\varphi)=\varphi \circ T$. By Theorem $4, T^{\sim}$ is interval preserving.

THEOREM 10. Let $E$ have the Riesz decomposition property. If $T: E \rightarrow G$ is interval preserving then $T^{\sim}: G^{\sim} \rightarrow E^{\sim}$ is an R-homomorphism.

Proof: For every $\varphi \in E^{\sim}, T^{\sim}(\varphi)=\varphi \circ T$. It follows from Theorem 8 that $T^{\sim}$ is an R-homomorphism.

We can now prove the result announced in the beginning of this paper as Theorem 1.

Proof of THEOREM 1: $T^{\sim}$ is interval preserving by Theorem 9. Also, $G^{\sim}$ is a Riesz space, and $E^{\sim}$ is an Archimedean, directed, partially ordered vector space. By Theorem 10, $T^{\sim \sim}$ is an R-homomorphism.

For the sake of clarity, we shall now choose another name for what was called a Riesz homomorphism in [3]. Let $F$ be a Riesz space. $T: E \rightarrow F$ is a weak R-homomorphism if for every $x, y \in E$ we have

$$
T(x) \vee T(y)=\inf \{T(z): z \in E \text { and } z \geqslant x, y\}
$$

We present an example of a weak R-homomorphism of which the biadjoint is not a weak R-homomorphism.

EXAMPLE. (A weak R-homomorphism of which the biadjoint is not a weak $R$-homomorphism.)

Let $H=\{f \in C[-1,1]: f(0)=(f(-1)+f(1)) / 2\}$ and let $i$ be the identity map from $H$ into $C[-1,1]$. Indeed, $i$ is a weak R-homomorphism. We shall show that the biadjoint of $i$ is not a weak R-homomorphism. Firstly, $H^{\sim}$ is Riesz isomorphic to $N=\left\{\mu \in C[-1,1]^{\sim}: \mu(\{0\})=0\right\}$. Although the latter result can be computed in a straightforward matter, we would like to point out the connection with barycentric maps (see $\left[9\right.$, Definition 2, p.188]). Indeed, the map $\rho:[-1,1] \rightarrow C[-1,1]^{\sim}$ defined by

$$
\rho(x)= \begin{cases}\delta_{x} & \text { if } x \in[-1,0) \cup(0,1] \\ \frac{1}{2}\left(\delta_{-1}+\delta_{1}\right) & \text { if } x=0\end{cases}
$$


is a barycentric map. It follows from a very general theorem about such barycentric maps (see [9, Theorem 6, p.188]) that the restriction map $j$ defined by $\left.\mu \mapsto \mu\right|_{H}$ from $N$ onto $H^{\sim}$ is a linear isometry. This linear isometry is positive, and thus it is a Riesz isomorphism from $N$ onto $H^{\sim}$ (see [10, Exercise 1.4.E5]).

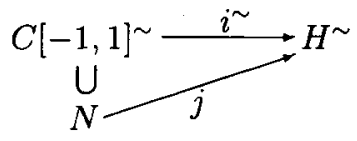

Now fix $\mu \in C[-1,1]^{\sim}$. Then there exists $\nu \in N$ with $j(\nu)=i^{\sim}(\mu)$, that is, $\nu(\{0\})=0$ and for every $f \in H, \int f d \mu=\int f d \nu$. By a straightforward application of the Dominated Convergence Theorem, we find that for all $f \in H$

$$
\begin{gathered}
f(-1)\left(\mu(\{-1\})+\frac{1}{2} \mu(\{0\})\right)+f(1)\left(\mu(\{1\})+\frac{1}{2} \mu(\{0\})\right)= \\
f(-1) \nu(\{-1\})+f(1) \nu(\{1\}) .
\end{gathered}
$$

By choosing $f \in H$ such that $f(-1)=0$ and $f(1)=2$, for example, $f(x)=x+1$ $(x \in[-1,1])$, we find

$$
\mu(\{1\})+\frac{1}{2} \mu(\{0\})=\nu(\{1\}) .
$$

Then

$$
\mu(\{-1\})+\frac{1}{2} \mu(\{0\})=\nu(\{-1\}) .
$$

We now show why $i^{\sim}$ is not interval preserving. Let $\mu=\delta_{-1}+2 \delta_{0}+3 \delta_{1}$. Then

(1) $\nu((-1,0) \cup(0,1))=0$,

(2) $\nu(\{-1\})=\mu(\{-1\})+\mu / 2(\{0\})$ and

(3) $\nu(\{1\})=\mu(\{1\})+\mu / 2(\{0\})$.

Thus, $\nu=2 \delta_{-1}+4 \delta_{1}$. Consider $\eta=4 \delta_{1}$ in $N$. Since $0 \leqslant \eta \leqslant \nu$ we have $0 \leqslant j(\eta) \leqslant$ $j(\nu)=i^{\sim}(\mu)$. Suppose $i^{\sim}$ is interval preserving. Then there exists $\bar{\eta} \in C[-1,1]^{\sim}$ with $0 \leqslant \bar{\eta} \leqslant \mu$ and $i^{\sim}(\bar{\eta})=j(\eta)$, that is, $\left.\bar{\eta}\right|_{H}=\left.4 \delta_{1}\right|_{H}$. It follows that $\bar{\eta}=a \delta_{-1}+b \delta_{0}+c \delta_{1}$ for some $a, b, c \geqslant 0$. But then, since $\eta(\{-1\})=0$, we have $a, b=0$. Therefore, $c=4$, and $\bar{\eta}=4 \delta_{1} \& \mu$. As a result $i^{\sim}$ is not interval preserving.

To prove that $i^{\sim \sim}$ is not a Riesz homomorphism, we suppose that it is. Then $i^{\sim \sim \sim}$ is interval preserving. Let $\mu \in C[-1,1]^{\sim}$ with $\mu \geqslant 0$, and $\nu \in H^{\sim}$ with $0 \leqslant \nu \leqslant i^{\sim}(\mu)$. Of course, $H^{\sim}$ and $C[-1,1]^{\sim}$ are Riesz subspaces of $H^{\sim \sim \sim}$ and $C[-1,1]^{\sim \sim \sim}$ respectively. Furthermore, since $C[-1,1]^{\sim}$ is an $L$-space, $C[-1,1]^{\sim}$ is an ideal in $C[-1,1]^{\sim \sim \sim}$ (see [10, p.68]). Therefore, $\mu \in C[-1,1]^{\sim \sim \sim}, \nu \in H^{\sim \sim \sim}$, and $i^{\sim}(\mu)=i^{\sim \sim \sim}(\mu) \in H^{\sim \sim \sim}$ with $\mu \geqslant 0$ and $0 \leqslant \nu \leqslant i^{\sim \sim \sim}(\mu)$. Since $i^{\sim \sim \sim}$ is interval preserving, there exists $\mu_{0} \in C[-1,1]^{\sim \sim \sim}$ with $0 \leqslant \mu_{0} \leqslant \mu$ and $i^{\sim \sim \sim}\left(\mu_{0}\right)=\nu$. Because $C[-1,1]^{\sim}$ is an ideal in $C[-1,1]^{\sim \sim \sim}$, 
$\mu_{0} \in C[-1,1]^{\sim}$. Thus, $i^{\sim}\left(\mu_{0}\right)=\nu$. But that implies $i^{\sim}$ is interval preserving. Therefore, $i^{\sim \sim}$ is not a Riesz homomorphism.

The above example augurs no hope for getting a result for weak R-homomorphisms in the style of Theorem 1. We are able to strengthen Theorem 1, by making a change in the definition of R-homomorphism. Indeed, if $F$ is a partially ordered vector space (that is, not necessarily a Riesz space) then a linear map $T: E \rightarrow F$ is called an $R$ homomorphism if $T$ considered as a map from $E$ to the enveloping Riesz space of $F$, denoted by $R[F]$, is an R-homomorphism in the sense defined in the introduction of this paper. We shall prove that the biadjoint of an $\mathrm{R}$-homomorphism with values in a space with the Riesz decomposition property is again an R-homomorphism. For the proof we need the observation that in Theorem 4 we can drop the condition that $G$ is a Riesz space.

Proposition 11. Let $F$ be a Dedekind complete Riesz space. If $T: E \rightarrow G$ is an $R$-homomorphism then the operator $S \mapsto S \circ T$ from $L_{r}(G, F)$ into $L_{r}(E, F)$ is interval preserving.

Proof: Since $T$ is an R-homomorphism, for every $x, y \in E^{+}$there exists $h \in E^{+}$ with $h \geqslant x, y$ and $T(h)=T(x) \vee T(y)$ in $R[G]$. The operator $S \mapsto S \circ T$ from $L_{r}(G, F)$ into $L_{r}(E, F)$ is positive. Assume that $S \in L_{r}(G, F)$ with $S \geqslant 0$, and let $V \in L_{r}(E, F)$ such that $0 \leqslant V \leqslant S \circ T$. We need to show the existence of $S_{1} \in L_{r}(G, F)$ with $0 \leqslant S_{1} \leqslant S$ and $S_{1} \circ T=V$.

By Kantorovič's Theorem, $S$ has a positive extension $S^{R} \in L_{r}(R[G], F)$. Diagrammatically we have:

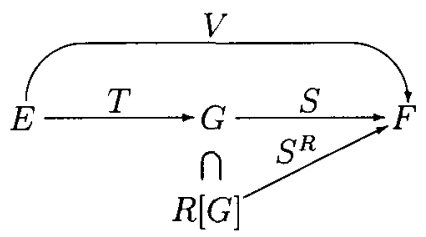

Since $\left.S^{R}\right|_{G}=S$ and $T(E) \subset G, S^{R} T=S T$. Hence, $V \leqslant S^{R} \circ T$. By Theorem 4, the operator $U \mapsto U \circ T$ from $L_{r}(R[G], F)$ into $L_{r}(E, F)$ is interval preserving. So, there exists $S_{1}^{R} \in L_{r}(R[G], F)$ with $0 \leqslant S_{1}^{R} \leqslant S^{R}$ and $S_{1}^{R} \circ T=V$.

Define $S_{1}=\left.S_{1}^{R}\right|_{G}$. $S_{1}$ is positive because $S_{1}^{R}$ is positive, and from $S_{1}^{R} \leqslant S^{R}$ it follows that $S_{1}=\left.S_{1}^{R}\right|_{G} \leqslant\left. S^{R}\right|_{G}=S$. Furthermore, $S_{1} \circ T=V$.

Corollary 12. If $T: E \rightarrow G$ is an R-homomorphism then $T^{\sim}: G^{\sim} \rightarrow E^{\sim}$ is interval preserving.

We now present the promised generalisation of Theorem 1 . 
THEOREM 13. Let $G$ have the Riesz decomposition property. If $T: E \rightarrow G$ is an $R$-homomorphism then $T^{\sim \sim}$ is an R-homomorphism $E^{\sim \sim} \rightarrow G^{\sim \sim}$.

Proof: By the above corollary, $T^{\sim}$ is interval preserving. $G^{\sim}$ is a Riesz space and $E^{\sim}$ is an Archimedean, directed, partially ordered vector space. Thus, Theorem 10 yields that $T^{\sim}$ is an R-homomorphism.

\section{REFERENCES}

[1] C.D. Aliprantis and O. Burkinshaw, Positive operators (Academic Press, Orlando, 1985).

[2] W. Arendt, Über das Spektrum regularen Operatoren, (Ph.D. Dissertation) (University of Tübingen, 1979).

[3] G. Buskes and A. van Rooij, 'The vector lattice cover of certain partially ordered groups', J. Austral. Math. Soc. 54 (1993), 352-367.

[4] G. Buskes and A. van Rooij, 'Hahn-Banach for Riesz homomorphisms', Indag. Math. 92 (1989), 25-34.

[5] P.F. Conrad, 'Minimal vector lattice covers', Bull. Austral. Math. Soc. 4 (1971), 35-39.

[6] F. Jellett, 'Homomorphisms and inverse limits of Choquet simplexes', Math. Z. 103 (1968), 219-226.

[7] L. Fuchs, Riesz vector spaces and Riesz algebras, Queen's Papers in Pure and Applied Mathematics 1 (Queens University, Kingston, On, 1966).

[8] J. Kim, 'The characterization of a lattice homomorphism', Canad J. Math. 27 (1975), $172-175$.

[9] H.E. Lacey, The isometric theory of classical Banach spaces (Springer-Verlag, Berlin, Heidelberg, New York, 1974).

[10] P. Meyer-Nieberg, Banach lattices (Springer-Verlag, Berlin, Heidelberg, New York, 1991).

11] A.L. Peressini, Ordered topological vector spaces (Harper and Row, New York, 1967).

[12] F. Takeo, 'On a simplex homomorphism', in Natural Science Report 34 (Ochanomizu University, 1983), pp. 53-59.

[13] F. Takeo, 'On a simplex homomorphism II', in Natural Science Report 35 (Ochanomizu University, 1984), pp. 47-56.

[14] A.W. Wickstead, 'The spectrum of an R-homomorphism', J. Austral. Math. Soc. 23 (1977), 42-45.

[15] A.C. Zaanen, Riesz spaces II (North-Holland, Amsterdam, 1983).

Department of Mathematics

The University of Mississippi

University MS 38677-9701

United States of America 